\title{
COMPARATIVE STUDY OF A FLEXIBLE AND RIGID TWO DEGREE OF FREEDOM HALF CAR MODEL
}

\author{
Saurav Talukdar', Anupam Mazumdar ${ }^{2}$, Karuna Kalita ${ }^{3}$ \\ ${ }^{1}$ Mechanical Engineering Department, Indian Institute of Technology Bombay, Powai, Mumbai - 400076, India \\ ${ }^{2}$ Civil Engineering Department, Indian Institute of Technology Guwahati, Guwahati - 781039, India \\ ${ }^{3}$ Mechanical Engineering Department, Indian Institute of Technology Guwahati, - 781039, karuna.kalita@iitg.ernet.in
}

\begin{abstract}
Ride quality is concerned with the feel of the passenger in the environment of a moving vehicle. It is one of the key indices in determining comfort levels of a vehicle. Although, "ride comfort" evaluation is subjective in nature, researchers have developed mathematical models to study and evaluate vehicle ride performance. Some popular models for vehicle ride analysis are - quarter car model, two dof (degree of freedom) and four dof half car model. These models model the chassis as a rigid body. This work removes this assumption and models the chassis of the vehicle as a flexible beam on a spring damper system at the front and rear using Euler beam theory. This elastic model has two dof-vehicle bounce and pitch, and has been compared with the rigid two dof model. Euler beam theory and Lagrangian mechanics are used to derive the equations of motion. Finite element method is used to validate this model. Experimental validation of the natural frequencies of this flexible beam is presented.
\end{abstract}

Keywords: Flexible ride model, Elastic ride model

\section{INTRODUCTION}

Research in the area of vehicle dynamics has progressively become more systematic and intensive since the early sixties. Mathematical modeling has become an important step to understand the underlying dynamics of the system. The development of computers has provided the computational power to have high fidelity models of real systems. Multibody dynamics (MBS) [1], Finite Element Method (FEM) [2] etc. are widely used in mechanical design and analysis.

Vehicle ride quality is considered to be one of the most important parameters to evaluate the performance of a vehicle. The designer has to achieve good ride comfort for the driver and passengers with acceptable control of body attitude and adequate control of dynamic tyre loads within the constraint of having a restricted amount of suspension working space available. These issues are classified as 'primary ride' and are studied with the help of mathematical models [3]. Some of the most popular and simple models are- quarter car model [4], two dof half car model [4], four dof half car model [5] and seven dof ride model [3]. These models consider the sprung masses and unsprung masses as rigid bodies. However, in reality the chassis is flexible and its flexibility needs to be considered to completely understand the dynamics. Vehicle ride models which have incorporated the flexible chassis are based on flexible multibody dynamics [6]. Finite element method is used to model the sprung mass as a flexible beam or as a flexible plate [7]. Some models use model reduction techniques to reduce the order of these models [8]. These models $[6,7,8]$ are based on numerical simulation and do not have a close form solution.

This paper presents an analytical model considering the sprung mass as a flexible beam on a spring damper system at the front and rear. A flexible beam system has infinite degrees of freedom. However, the variables of interest in this context are - the overall translation and rotation of the beam. Hence, this model also has two degrees of freedom - bounce and pitch like the conventional two dof half car model [4], which is the rigid body counterpart. Euler beam theory [9] is used to model the beam and Lagrangian mechanics [10] along with the concept of tracking frame [11] is used to derive the equations of motion. The conventional two dof model will be referred to as 'rigid two dof model' and model developed in this paper will be referred to as 'flexible two dof model'. The results are validated by finite element and experimental results. A comparison between both the models in time domain and frequency domain is presented and the differences are discussed. The paper is organized as follows: Section 1 contains the vehicle models. Finite element modeling of a flexible beam is explained in Section 2. Section 3 presents the results and discussion, followed by summary in Section 4.

\section{VEHICLE MODELS}

The two vehicle models compared in this paper viz. the rigid two dof model and the flexible two dof model, which are presented below. 


\section{RIGID TWO DOF MODEL}

A schematic of the rigid two dof model is shown in Figure 1. This model does not consider the sprung mass and unsprung mass to be distinct. It has one combined mass $\left(m_{s}\right)$ which represents half of a vehicle supported on front $\left(k_{1}\right.$ and $c_{1}$ are the front suspension stiffness and damping respectively) and rear suspension ( $k_{2}$ and $c_{2}$ are the rear suspension stiffness and damping respectively). The springs and dampers are considered to be at the extreme ends. The two degrees of freedom are - vertical displacement of the chassis $\left(x_{s}\right)$ and the pitching motion $(\varphi)$. The distance of the centre of gravity (CG) from the front $\left(l_{1}\right)$ and rear axle $\left(l_{2}\right)$ are used to write the displacements of the front and rear end as well as moment arm for the moment equation ( $J$ is the moment of inertia). The inputs to this model are the road excitations at the front $\left(x_{r f}\right)$ and rear $\left(x_{r r}\right)$. The main assumptions of this model are-
a) Unsprung mass is not considered.
b) Sprung mass is considered to be a rigid body.
c) The linear suspension spring and viscous damping is assumed.
d) Springs and dampers are considered to be at the ends.
e) Small pitch displacement.

The equations of motion are -

$\mathbf{M} \ddot{\mathbf{x}}+\mathbf{C} \dot{\mathbf{x}}+\mathbf{K} \mathbf{x}=\mathbf{F}_{1} \mathbf{u}_{1}+\mathbf{F}_{2} \mathbf{u}_{2}$

where,

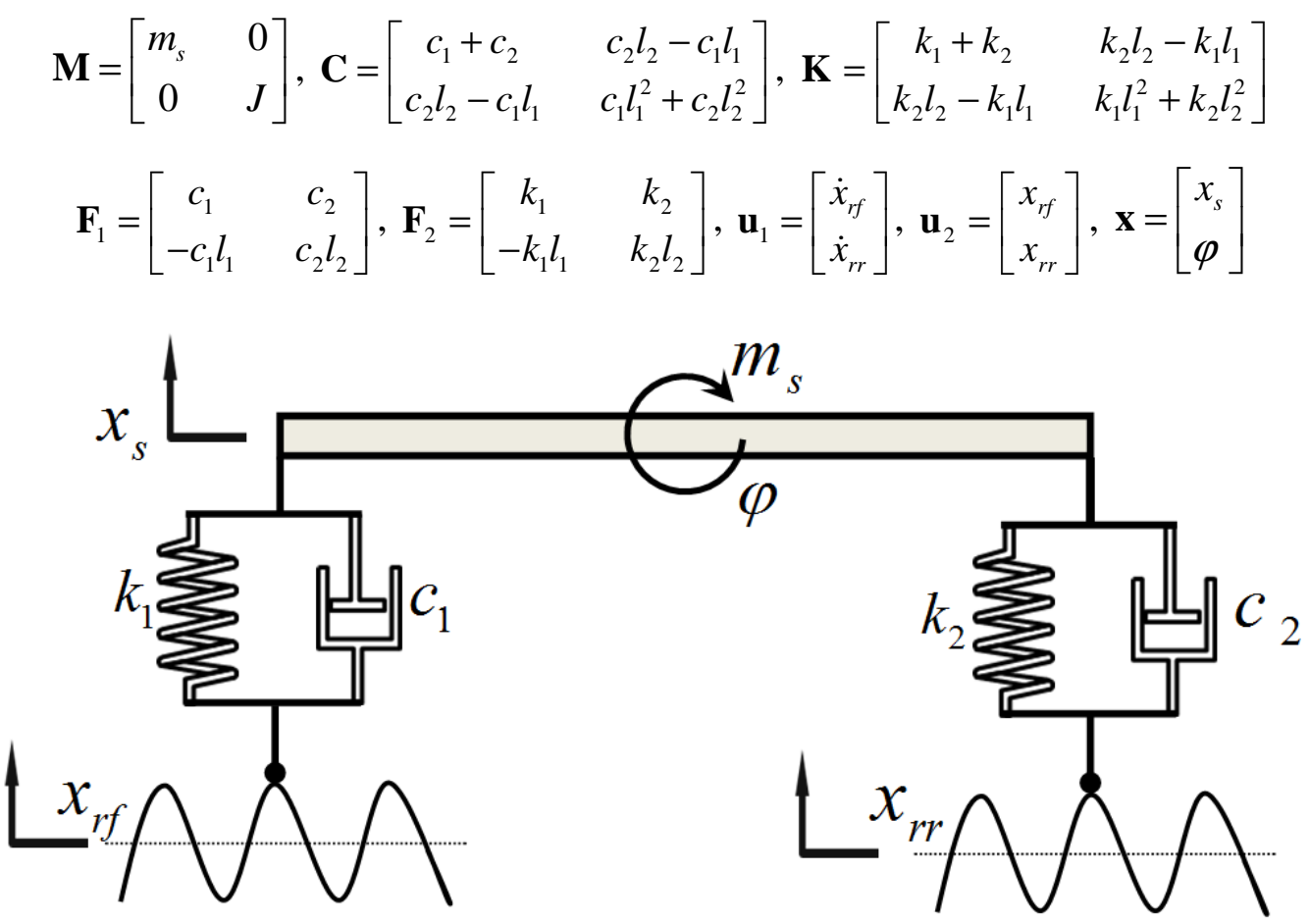

Figure1. Rigid two dof half car model

\section{FLEXIBLE TWO DOF MODEL}

A schematic of the flexible two dof model is shown in Figure 2. This model considers the sprung mass as an Euler beam supported on a spring damper system at the front and rear end of the beam. The main assumptions of this model are -

a) Unsprung mass is not considered.

b) The beam is considered to undergo planar motion and there is only in-plane bending.

c) The beam is made of homogeneous material. d) Linear suspension spring and viscous damping is assumed.

e) Small pitch displacement.

f) The extension of the spring due to the different vibration modes has been neglected. The expression for potential energy of the spring is written considering the rigid body mode of vibration only.

In Figure 2, the coordinate system $\boldsymbol{n}$ is an inertial reference frame whereas the coordinate system $\boldsymbol{b}$ is a body fixed frame system. 


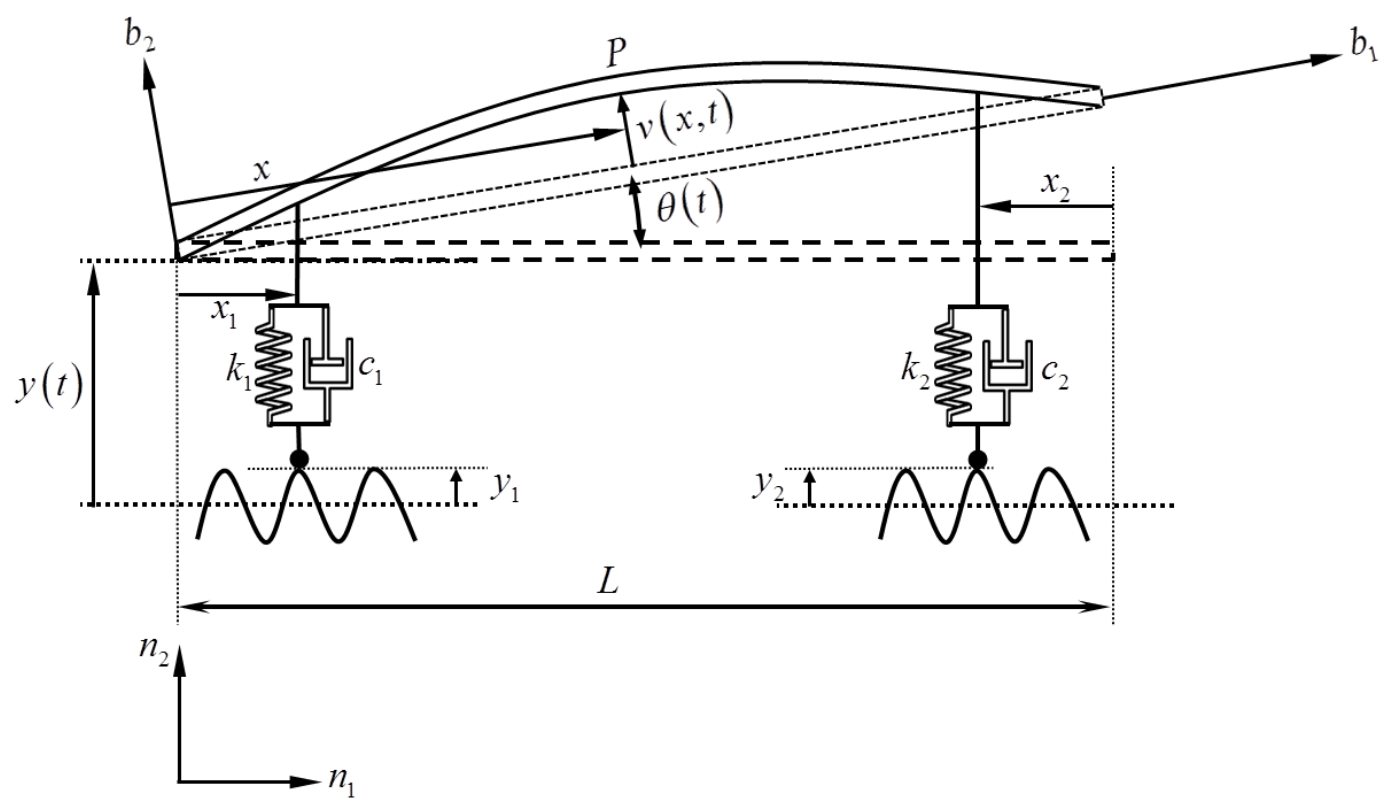

Figure 2. Flexible two dof half car model

Position vector $(R)$ of any point $\boldsymbol{P}$ can be written as

$\mathbf{R}(x, y, t)=\mathbf{R}_{\mathbf{0}}(y)+\mathbf{r}(x, t)=y(t) \hat{n}_{2}+x \hat{b}_{1}+v(x, t) \hat{b}_{2}$

The velocity of point $\boldsymbol{P}$ with respect to the inertial frame can be written as

$\dot{\mathbf{R}}(x, y, t)=\dot{y}(t) \hat{n}_{2}+\dot{v} \hat{b}_{2}+\dot{\theta} \hat{b}_{3} \times\left\{x \hat{b}_{1}+v(x, t) \hat{b}_{2}\right\}$

$\dot{\mathbf{R}}(x, y, t)=\dot{y}(t) \hat{n}_{2}+(\dot{v}+x \dot{\theta}) \hat{b}_{2}-\dot{\theta} v \hat{b}_{1}$

The unit vector in the inertial frame is related to the body fixed frame by

$\left[\begin{array}{ccc}\cos \theta & -\sin \theta & 0 \\ \sin \theta & \cos \theta & 0 \\ 0 & 0 & 1\end{array}\right]\left[\begin{array}{l}\hat{b}_{1} \\ \hat{b}_{2} \\ \hat{b}_{3}\end{array}\right]=\left[\begin{array}{l}\hat{n}_{1} \\ \hat{n}_{2} \\ \hat{n}_{3}\end{array}\right]$

Using small angle approximation $\sin \theta \approx \theta, \cos \theta \approx 1$ and neglecting the nonlinear terms the velocity of point $P$ in the body frame is given by equation (3).

$\dot{\mathbf{R}}=(\dot{y}+\dot{v}+\dot{\theta} x) \hat{b}_{2}$

The kinetic energy of the beam $(T)$ can be expressed as

$$
\begin{aligned}
T & =\frac{1}{2} \rho A \int_{L}\left(\dot{y}^{2}+\dot{v}^{2}+x^{2} \dot{\theta}^{2}+2 \dot{\theta} \dot{v} x+2 \dot{v} \dot{y}+2 \dot{y} \dot{\theta} x\right) \\
& =\frac{1}{2} m \dot{y}^{2}+J_{0} \dot{\theta}^{2}+\frac{1}{2} \rho A \int_{L} \dot{v}^{2} d x+\rho A \dot{\theta} \int_{L} \dot{v} x d x+\rho A \dot{y} \int_{L} \dot{v} d x+\frac{1}{2} m \dot{y} L \dot{\theta}
\end{aligned}
$$

where $m$ is the mass of the beam and $J_{0}=\frac{1}{3} m L^{2}$ is the moment of inertia of the beam. Here $y$ and $\theta$ are the function of time only whereas $v$ is a function of both position and time. The potential energy of the beam $(U)$ with the spring and damper system is formulated. The potential energy of the system has contributions from both the springs and strain energy of the beam. The potential energy of the system is given by - 
$U=\frac{1}{2} k_{1}\left(y+x_{1} \theta-y_{1}\right)^{2}+\frac{1}{2} k_{2}\left\{y+\left(L-x_{2}\right) \theta-y_{2}\right\}^{2}+E I \int_{L} v^{\prime \prime} d x$

According to the Extended Hamiltonian principal [10],

$\int \delta L d t+\int \delta W_{n c} d t=0$

where, $L$ is the Lagrangian and $W_{n c}$ is the non-conservative work [10] and $L=T-U$. The Rayleigh term, $R$ is given by [10] as

$R=\frac{1}{2} c_{1}\left(\dot{y}+x_{1} \dot{\theta}-\dot{y}_{1}\right)^{2}+\frac{1}{2} c_{2}\left(\dot{y}+\left(L-x_{2}\right) \dot{\theta}-\dot{y}_{2}\right)^{2}$

Using Lagrange's equation,

$\frac{d}{d t}\left(\frac{\partial T}{\partial \dot{q}}\right)-\frac{\partial T}{\partial q}+\frac{\partial U}{\partial q}+\frac{\partial R}{\partial \dot{q}}=0$

where, $q$ is the generalized coordinate. The generalised coordinates for this system are - $y, \theta, v$. Using these generalised coordinates and equation (7), the governing dynamical equations of the system under consideration is given by equation (8) to (10).

$$
\begin{aligned}
& m \ddot{y}+\rho A \int_{L} \ddot{v} d x+\frac{1}{2} m L \ddot{\theta}+k_{1}\left(y+x_{1} \theta\right) \\
& \left.+k_{2}\left(y+\left(L-x_{2}\right) \theta\right)+c_{1}\left(\dot{y}+x_{1} \dot{\theta}\right)+c_{2}\left(\dot{y}+\left(L-x_{2}\right) \dot{\theta}\right)\right\} \\
& =k_{1} y_{1}+k_{2} y_{2}+c_{1} \dot{y}_{1}+c_{2} \dot{y}_{2} \\
& \left.\begin{array}{l}
J_{0} \ddot{\theta}+\rho A \int_{L} \ddot{v} x d x+\frac{1}{2} m L \ddot{y}+k_{1} x_{1}\left(y+x_{1} \theta\right)+k_{2}\left(L-x_{2}\right) y+k_{2}\left(L-x_{2}\right)^{2} \theta+c_{2} L \dot{y}+c_{2} L^{2} \dot{\theta}= \\
k_{1} x_{1} y_{1}+c_{1} x_{1} \dot{y}_{1}+k_{2}\left(L-x_{2}\right) y_{2}+c_{2}\left(L-x_{2}\right) \dot{y}_{2}
\end{array}\right\} \\
& \rho A \ddot{v}+\rho A(x \ddot{\theta}+\ddot{y})+E I \frac{\partial^{4} v}{\partial x^{4}}=0
\end{aligned}
$$

$k_{1}, k_{2}, c_{1}$, and $c_{2}$ are the stiffness and damping coefficients of front and rear springs and dampers respectively. The consequence of assumption (f) of this model is that it gives accurate results for $x_{1}=0$ and $x_{2}=0$. Hence, the spring and damper system has to be attached at the ends. However, $x_{1}$ and $x_{2}$ have been retained in the derivation even if they are taken to be zero in the simulations so as to explain the derivation for a general location of springs. If $x_{1} \neq 0 \& x_{2} \neq 0$, the problem can be formulated again with slight modification of the potential energy expression (equation (5)) and Rayleigh term (equation (6)). The modified potential energy expression for general spring-damper location is -

$U=\frac{1}{2} k_{1}\left(y+x_{1} \theta+v-y_{1}\right)^{2}+\frac{1}{2} k_{2}\left\{y+\left(L-x_{2}\right) \theta+v-y_{2}\right\}^{2}+E I \int_{L} v^{\prime \prime} d x$

The modified Rayleigh term is-

$$
R=\frac{1}{2} c_{1}\left(\dot{y}+x_{1} \dot{\theta}+\dot{v}-\dot{y}_{1}\right)^{2}+\frac{1}{2} c_{2}\left(\dot{y}+\left(L-x_{2}\right) \dot{\theta}+\dot{v}-\dot{y}_{2}\right)^{2}
$$

Using equation (4), (11), (12) and (7), the new set of governing equations of motion can be obtained. Henceforth, this work would consider the case of $x_{1}=0$ and $x_{2}=0$.

The natural frequencies and mode shapes are obtained by solving the free vibration problem, hence, the damping and base excitations are not considered. The damping is neglected as the quantities of interest are the undamped natural frequency and its corresponding mode shapes. To determine the mode shapes and natural frequencies, let

$v(x, t)=V(x) \exp (j \omega t)$ 


$$
\begin{aligned}
& \theta(t)=\Theta \exp (j \omega t) \\
& y(t)=Y \exp (j \omega t)
\end{aligned}
$$

Using equations (13) to (15) in (8) to (10), neglecting the damping and base excitation along with the substitution,

$W(x)=V(x)+x \Theta$

the equations are-

$\left(k_{1}+k_{2}-m \omega^{2}\right) Y-\rho A \omega^{2} \int_{L} W d x+\Theta\left\{k_{1} x_{1}+k_{2}\left(L-x_{2}\right)\right\}=0$

$-\rho A \omega^{2} \int_{L} x W d x+Y\left\{k_{1} x_{1}+k_{2}\left(L-x_{2}\right)-\frac{1}{2} m L \omega^{2}\right\}+\Theta\left\{k_{1} x_{1}^{2}+k_{2}\left(L-x_{2}\right)^{2}\right\}=0$

$-\rho A \omega^{2} W-\rho A \omega^{2} Y+E I W^{i v}=0$

Substituting $\int_{L} W d x=\alpha$ in equation (16),

$Y=\frac{\rho A \omega^{2} \alpha-\Theta\left\{k_{1} x_{1}+k_{2}\left(L-x_{2}\right)\right\}}{k_{1}+k_{2}-m \omega^{2}}$

Using equation (19) in (18),

$E I W^{i v}-\rho A \omega^{2} W=\rho A \omega^{2}\left(\frac{\rho A \omega^{2} \alpha-\Theta\left\{k_{1} x_{1}+k_{2}\left(L-x_{2}\right)\right\}}{k_{1}+k_{2}-m \omega^{2}}\right)=\lambda \alpha+\psi \Theta$

where, $\lambda=\frac{\left(\rho A \omega^{2}\right)^{2}}{k_{1}+k_{2}-m \omega^{2}}$ and $\psi=\frac{\rho A \omega^{2}\left(k_{1} x_{1}+k_{2}\left(L-x_{2}\right)\right)}{k_{1}+k_{2}-m \omega^{2}}$.

The general solution of equation (20) can be written as,

$W(x)=a_{1} \cos k x+a_{2} \sin k x+a_{3} \cosh k x+a_{4} \sinh k x-B \alpha+D \theta$

where, $k=\sqrt[4]{\frac{\rho A}{E I}} \sqrt{\omega}, B=\frac{\rho A \omega^{2}}{k_{1}+k_{2}-m \omega^{2}}, D=\frac{k_{1} x_{1}+k_{2}\left(L-x_{2}\right)}{k_{1}+k_{2}-m \omega^{2}}$

Substituting the following expression in equation (17) and using equation (19),

$\int_{L} x W(x) d x=\beta$

$\Theta=\frac{\rho A \omega^{2} \beta-\left\{k_{1} x_{1}+k_{2}\left(L-x_{2}\right)-\frac{1}{2} m L \omega^{2}\right\} B \alpha}{k_{1} x_{1}^{2}+k_{2}\left(L-x_{2}\right)^{2}-D\left(k_{1} x_{1}+k_{2}\left(L-x_{2}\right)-\frac{1}{2} m L \omega^{2}\right)}=m_{1} \beta-m_{2} \alpha$

where, $\quad\left\{\begin{array}{l}m_{1}=\frac{\rho A \omega^{2}}{k_{1} x_{1}^{2}+k_{2}\left(L-x_{2}\right)^{2}-D\left(k_{1} x_{1}+k_{2}\left(L-x_{2}\right)-\frac{1}{2} m L \omega^{2}\right)} \\ m_{2}=\frac{\left\{k_{1} x_{1}+k_{2}\left(L-x_{2}\right)-\frac{1}{2} m L \omega^{2}\right\} B}{k_{1} x_{1}^{2}+k_{2}\left(L-x_{2}\right)^{2}-D\left(k_{1} x_{1}+k_{2}\left(L-x_{2}\right)-\frac{1}{2} m L \omega^{2}\right)}\end{array}\right.$ 
The boundary conditions used to solve this problem are

1. The choice of the body fixed frame $\boldsymbol{b}$ gives $W(0)=0$ and $W(L)=L \Theta$

2. The bending moment at both the ends is zero, which implies

$$
\frac{d^{2} W(0)}{d x^{2}}=0 \text { and } \frac{d^{2} W(L)}{d x^{2}}=0
$$

Using these four conditions and substituting equation (21) in the definitions of $\alpha$ and $\beta$, a system of six homogenous equations with $\mathbf{x}=\left[\begin{array}{llllll}a_{1} & a_{2} & a_{3} & a_{4} & \alpha & \beta\end{array}\right]^{T}$ as unknowns can be obtained as

$\mathbf{D} \mathbf{x}=\mathbf{0}$

where, $\mathbf{D}$ is a $6 \times 6$ matrix described below. The detailed steps are

Using $W(0)=0$ in equation $(21)$,

$a_{1}+a_{3}-\left(B+D m_{2}\right) \alpha+D m_{1} \beta=0$

Using $W(L)=L \Theta$ in equation $(21)$,

$a_{1} \cos k L+a_{2} \sin k L+a_{3} \cosh k L+a_{4} \sinh k L-\left\{B+(D-L) m_{2}\right\} \alpha+(D-L) m_{1} \beta=0$

Using $E I W^{\prime \prime}(0)=0$ in equation (21),

$a_{1}-a_{3}=0$

Using $E I W^{\prime \prime}(L)=0$ in equation (21),

$-a_{1} \cos k L-a_{2} \sin k L+a_{3} \cosh k L+a_{4} \sinh k L=0$

Using equation (21) in definition of $\alpha$,

$a_{1} \sin k L-a_{2}(\cos k L-1)+a_{3} \sinh k L+a_{4}(\cosh k L-1)-k(1+B L) \alpha+k D L \theta=0$

or, $a_{1} \sin k L-a_{2}(\cos k L-1)+a_{3} \sinh k L+a_{4}(\cosh k L-1)-k\left(1+B L+m_{2} D L\right) \alpha+m_{1} k D L \beta=0$

Using equation (21) in definition of $\beta$,

$a_{1}(k L \sin k L+\cos k L-1)+a_{2}(\sin k L-k L \cos k L)+$

$a_{3}(k L \sinh k L-\cosh k L+1)+a_{4}(k L \cosh k L-\sinh k L)-$

$\frac{1}{2} B k^{2} L^{2} \alpha-k^{2} \beta+\frac{1}{2} D k^{2} L^{2} \theta=0$

or, $a_{1}(k L \sin k L+\cos k L-1)+a_{2}(\sin k L-k L \cos k L)+$

$a_{3}(k L \sinh k L-\cosh k L+1)+a_{4}(k L \cosh k L-\sinh k L)-$

$\frac{1}{2}\left(B k^{2} L^{2}+D k^{2} L^{2} m_{2}\right) \alpha+\left(\frac{1}{2} D k^{2} L^{2} m_{1}-k^{2}\right) \beta=0$

Equations (24) to (29) can be written in the form of equation (23) where,

$\mathbf{D}=\left[\begin{array}{cccccc}1 & 0 & 1 & 0 & -\left(B+D m_{2}\right) & D m_{1} \\ \cos k L & \sin k L & \cosh k L & \sinh k L & -\left\{B+(D-L) m_{2}\right\} & (D-L) m_{1} \\ 1 & 0 & -1 & 0 & 0 & 0 \\ -\cos k L & -\sin k L & \cosh k L & \sinh k L & 0 & 0 \\ \sin k L & 1-\cos k L & \sinh k L & \cosh k L-1 & -k\left(1+B L+m_{2} D L\right) & m_{1} K D L \\ \left(\begin{array}{c}k L \sin k L \\ +\cos k L-1\end{array}\right) & \left(\begin{array}{l}\sin k L- \\ k L \cos k L\end{array}\right) & \left(\begin{array}{l}k L \sinh k L- \\ \cosh k L+1\end{array}\right) & \left(\begin{array}{l}k L \cosh k L \\ -\sinh k L\end{array}\right) & -\frac{k^{2} L^{2}}{2}\left(B+D m_{2}\right) & -k^{2}+\frac{k^{2} L^{2} D m_{1}}{2}\end{array}\right]$ 


\section{$\mathbf{x}=\left[\begin{array}{llllll}a_{1} & a_{2} & a_{3} & a_{4} & \alpha & \beta\end{array}\right]^{T}$}

To ensure non trivial solutions the determinant of the coefficient matrix $\mathbf{D}$ has to be zero. This gives the values of $k$ corresponding to the natural angular frequencies of the system. Using these values of $k$ and assuming $a_{1}=1$, the other unknowns in $\mathbf{x}$ can be obtained, which can then be used to compute the mode shapes using equation (30).

$V(x)=W(x)-x \Theta$

$(W(x)$ in equation (30) could be be obtained from equation (21))

The obtained mode shape $V(\mathrm{x})$ will be used to solve the forced vibration problem i.e., the system with spring, damper and base excitation. Using separation of variables the displacement of the beam as a function of space and time in the body fixed coordinate system is given by equation (31).

$v(x, t)=V(x) \eta(t)$

Substituting equation (31) in equation (8) and (9),

$\left.\begin{array}{l}m \ddot{y}+\rho A \ddot{\eta} \int_{L} V(x) d x+\frac{1}{2} m L \ddot{\theta}+k_{1}\left(y+x_{1} \theta\right)+k_{2}\left\{y+\theta\left(L-x_{2}\right)\right\}+ \\ c_{1}\left(\dot{y}+x_{1} \dot{\theta}\right)+c_{2}\left\{\dot{y}+\left(L-x_{2}\right) \dot{\theta}\right\}=k_{1} y_{1}+k_{2} y_{2}+c_{1} \dot{y}_{1}+c_{2} \dot{y}_{2}\end{array}\right\}$

$J_{0} \ddot{\theta}+\rho A \ddot{\eta} \int_{L} V(x) x d x+\frac{1}{2} m L \ddot{y}+k_{1} x_{1}\left(y+x_{1} \theta\right)+$

$k_{2}\left(L-x_{2}\right) y+k_{2}\left(L-x_{2}\right)^{2} \theta+c_{2} L \dot{y}+c_{2} L^{2} \dot{\theta}=$

$k_{1} x_{1} y_{1}+c_{1} x_{1} \dot{y}_{1}+k_{2}\left(L-x_{2}\right) y_{2}+c_{2}\left(L-x_{2}\right) \dot{y}_{2}$

Using equation (31) in (10), multiplying both sides by $W(x)$ and integrating between 0 to $L$,

$\left.\begin{array}{l}\rho A \ddot{\eta} \int_{L} V(x) W(x) d x+\rho A \ddot{\theta} \int_{L} x W(x) d x+ \\ \rho A \ddot{y} \int_{L} W(x) d x+E I \eta \int_{L} W(x) \frac{d^{4} V(x)}{d x^{4}} d x=0\end{array}\right\}$

The equations (32) to(34) can be re-written in the standard form as in equation (1).

$\mathbf{M} \ddot{\mathbf{x}}+\mathbf{C} \dot{\mathbf{x}}+\mathbf{K} \mathbf{x}=\mathbf{F}_{1} \mathbf{u}_{1}+\mathbf{F}_{2} \mathbf{u}_{2}$

where,

$\mathbf{x}=\left[\begin{array}{c}y \\ \theta \\ \eta\end{array}\right], \mathbf{u}_{1}=\left[\begin{array}{c}\dot{y}_{1} \\ \dot{y}_{2}\end{array}\right], \mathbf{u}_{2}=\left[\begin{array}{l}y_{1} \\ y_{2}\end{array}\right]$

$\mathbf{M}=\left[\begin{array}{ccc}m & 0.5 m L & \rho A \int_{L} V(x) d x \\ 0.5 m L & J_{0} & \rho A \int_{L} x V(x) d x \\ \rho A \int_{L} W(x) d x & \rho A \int_{L} x W(x) d x & \rho A \int_{L} V(x) W(x) d x\end{array}\right]$ 
$\mathbf{C}=\left[\begin{array}{ccc}c_{1}+c_{2} & c_{1} x_{1}+c_{2}\left(L-x_{2}\right) & 0 \\ c_{2} L & c_{2} L^{2} & 0 \\ 0 & 0 & 0\end{array}\right]$

$\mathbf{K}=\left[\begin{array}{ccc}k_{1}+k_{2} & k_{1} x_{1}+k_{2}\left(L-x_{2}\right) & 0 \\ k_{1} x_{1}-k_{2} x_{2} & k_{1} x_{1}^{2}+k_{2}\left(L-x_{2}\right)^{2} & 0 \\ 0 & 0 & E I \int_{L} W(x) \frac{d^{4} V(x)}{d x^{4}} d x\end{array}\right]$

$\mathbf{F}_{1}=\left[\begin{array}{cc}c_{1} & c_{2} \\ c_{1} x_{1} & c_{2}\left(L-x_{2}\right) \\ 0 & 0\end{array}\right], \mathbf{F}_{2}=\left[\begin{array}{cc}k_{1} & k_{2} \\ k_{1} x_{1} & k_{2}\left(L-x_{2}\right) \\ 0 & 0\end{array}\right]$

Each mode shape obtained from equation (30) can be used in equation (32) to (34) along with numerical integration techniques to obtain the corresponding time response function $\eta_{i}(t)$, bounce displacement of $\mathrm{CG}\left(y_{i}(t)+\frac{L}{2} \theta_{i}(t)\right)$ and pitch displacement of CG $\theta_{i}(t)$.The general response of the beam in the body fixed coordinate system is given by

$v(x, t)=\sum_{i=0}^{\infty} V_{i}(x) \eta_{i}(t)$

where, $V_{i}(x)$ is the mode shape corresponding to the $i^{\text {th }}$ natural frequency and $\eta_{i}(t)$ is the time response of the beam corresponding to the $i^{\text {th }}$ mode. The net bounce displacement and pitch displacement of the CG is the summation due to each mode and can be computed using equation (36) and (37).

$\bar{y}(t)=\sum_{i=1}^{\infty}\left(y_{i}(t)+\frac{L}{2} \theta_{i}(t)\right)$

$\bar{\theta}(t)=\sum_{i=1}^{\infty} \theta_{i}(t)$

Equations (35) to (37) along with (32) to (34) and initial conditions provide a solution to the forced excitation problem.

\section{FINITE ELEMENT MODELING}

Finite element method has been used to calculate the natural frequency and its associated modes of the flexible beam supported by two springs at the both ends. The damping is not considered for finite element analysis. The elemental equations of stiffness and mass matrices have been derived for bending motion of the beam assuming Euler-Bernoulli bending theory [13]. Figure 3 shows slender beam supported by two springs at both ends. The beam is discretized with $(N-1)$ two noded elements generating $N$ nodes. Figure 4 shows any arbitrary element with the two degrees of freedom per node. In Figure $4, v_{i}$ and $v_{i+1}$ represents the displacements of $i{ }^{\text {th }}$ and $(i+1)^{\text {th }}$ node respectively and $\varphi_{i}$ and $\varphi_{i+1}$ represents the deflections of $i^{\text {th }}$ and $(i+1)^{\text {th }}$ node respectively.

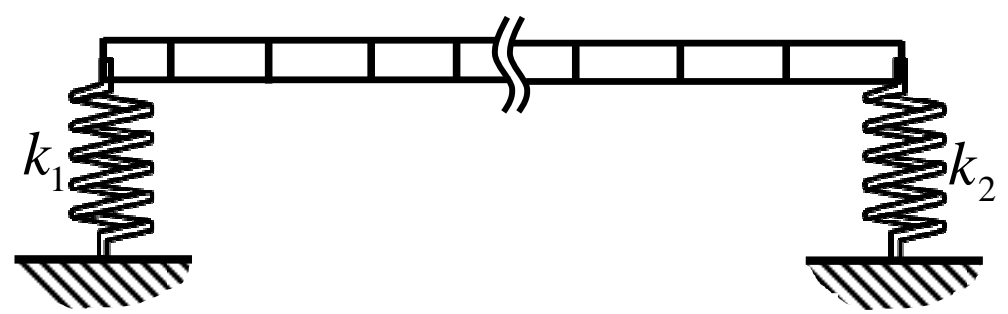

Figure 3. Finite element model 


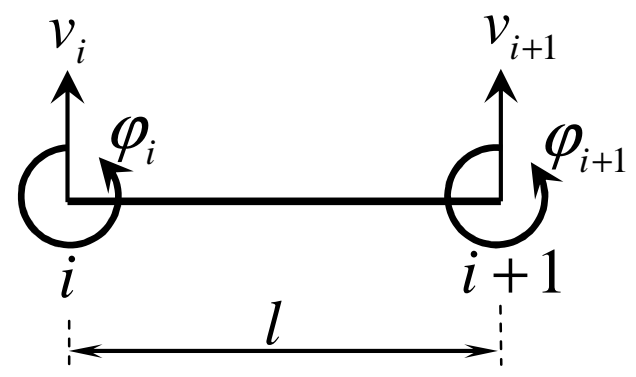

Figure 4. One finite element

The elemental stiffness and mass matrices are shown in equations (38) and (39) respectively.

$$
\mathbf{M}^{e}=\frac{\rho A l}{420}\left[\begin{array}{cccc}
156 & 22 l & 54 & -13 l \\
22 l & 4 l^{2} & -13 l & -3 l^{2} \\
54 & -13 l & 156 & -22 l \\
-13 l & -3 l^{2} & -22 l & 4 l^{2}
\end{array}\right]
$$

where $\rho$ is the density, $A$ is the cross sectional area and the $l$ is the length of the finite element.

$$
\mathbf{K}^{e}=\frac{E I}{l^{3}}\left[\begin{array}{cccc}
12 & 6 l & -12 & 6 l \\
6 l & 4 l^{2} & -6 l & 2 l^{2} \\
-12 & -6 l & 12 & -6 l \\
6 l & 2 l^{2} & -6 l & 4 l^{2}
\end{array}\right]
$$

where $E$ is the Young's modulus, $I$ is the flexural moment of inertia and the $l$ is the length of the finite element.

The elemental spring and mass matrices of each element are derived and assembled to give the complete equation of motion. The complete equation of motion can be written as

$\mathbf{M}_{G} \ddot{q}_{G}+\mathbf{K}_{G} q_{G}=\mathbf{0}$

where $\mathbf{M}_{G}$ is the global mass matrix whose size is $(2 N \times 2 N), \mathbf{K}_{G}$ is the global stiffness matrix whose size is $(2 N \times 2 N)$ and $q_{G}$ is the nodal displacements whose size is $(2 N \times 1)$. The boundary conditions have been applied by incorporating the spring stiffness at the $1^{\text {st }}$ and $N^{\text {th }}$ nodes. The eigenvalue and eigenvectors are calculated using the 'eig' command of MATLAB ${ }^{\circledR}$ as follows:

$[$ eval, evec $]=\operatorname{eig}\left(\mathbf{K}_{G}, \mathbf{M}_{G}\right)$

where $e v a l$ gives the squares of the natural frequencies and evac gives the corresponding mode shapes.

\section{RESULTS AND DISCUSSION}

A comparison between the rigid and the flexible models described in the previous sections is presented here. The numerical parameters used for the simulation are tabulated (see Table 3 and Table 4) in the appendix. A MATLAB code has been written to calculate the natural frequencies and the mode shapes for the flexible two dof model. The natural frequencies and mode shapes obtained from the analytical solutions are validated using finite element analysis. The beam is modeled as an Euler-Bernoulli beam. A total number of 100 elements are considered with two degrees of freedom per node. The first four modes of the flexible two dof model are used to calculate the time response of the beam. The first four natural frequencies obtained from the analytical solution of flexible two dof model and FEM model is tabulated in Table 1.

Table 1. Natural frequencies (in $\mathrm{Hz}$ ) of the flexible two dof model from analytical and FEM approach.

\begin{tabular}{|l|c|c|c|c|}
\hline \multicolumn{1}{|c|}{ Model } & $\omega_{1}$ & $\omega_{2}$ & $\omega_{3}$ & $\omega_{4}$ \\
\hline Analytical & 10.4837 & 18.2553 & 243.8926 & 670.1111 \\
\hline FEM & 10.4861 & 18.2404 & 243.8914 & 670.1108 \\
\hline
\end{tabular}

A small scale experimental setup has been built to verify the natural frequencies obtained from the analytical model. The setup consists of a mild steel beam on two springs of equal 
stiffness at the ends. The parameters of this setup are listed in the appendix (see Table 5). The beam is excited with an impulse hammer and two accelerometers are mounted at two points on the beam to capture the response. The impulse hammer and accelerometers are connected to a Data Acquisition System. A picture of the experimental setup is shown in Figure 3. The first four natural angular frequencies of this beam obtained from the analytical model, FEM and experiment are listed in Table 2.

Table 2. Natural angular frequencies (in $\mathrm{rad} / \mathrm{s}$ ) of the experimental beam from analytical model, FEM model and experiment

\begin{tabular}{|l|l|l|l|l|}
\hline \multicolumn{1}{|c|}{ Model } & \multicolumn{1}{c|}{$\omega_{1}$} & \multicolumn{1}{c|}{$\omega_{2}$} & \multicolumn{1}{c|}{$\omega_{3}$} & \multicolumn{1}{c|}{$\omega_{4}$} \\
\hline Analytical & 66.892 & 185.537 & 311.019 & 549.781 \\
\hline FEM & 66.892 & 185.538 & 311.018 & 549.783 \\
\hline Experiment & 69.12 & 182.20 & 314.11 & 552.34 \\
\hline
\end{tabular}

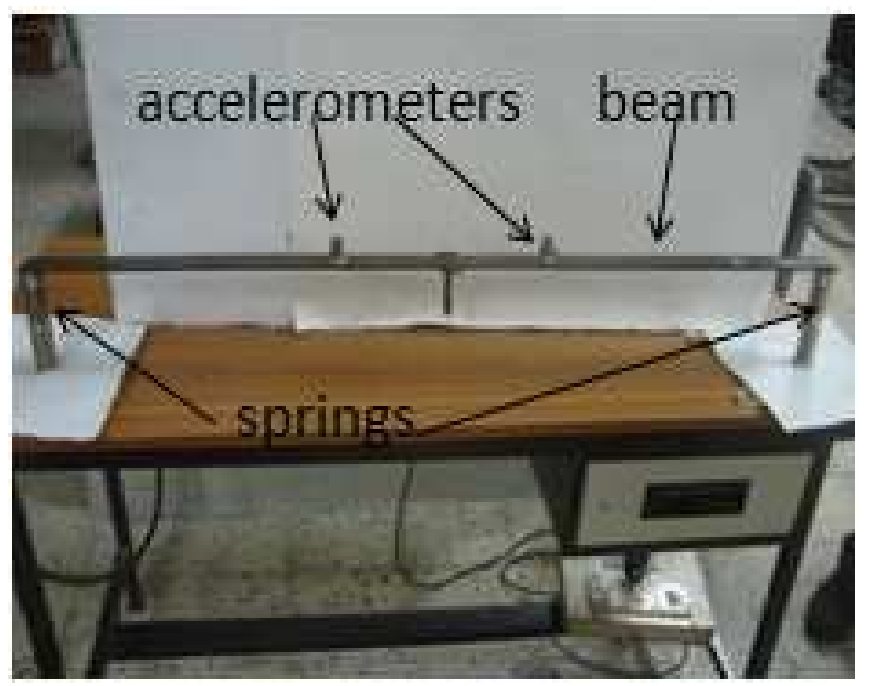

Figure 3. Experimental setup to verify natural frequencies

Figure 4 shows the acceleration frequency response function (FRF) obtained from the experimental setup. Peaks can be observed in this experimentally obtained FRF for frequencies close to the natural frequencies predicted by the analytical model. This is supportive of the analytical solution described in the previous section. However, peaks are also seen for frequencies which are not the natural frequencies e.g., 150 $\mathrm{rad} / \mathrm{s}, 402 \mathrm{rad} / \mathrm{s}$ etc. These could be due to the unaccounted structural damping of the system, the cylindrical rods welded at the end part of the beam to hold the springs, non-uniformity of beam etc.

The mode shapes of the beam have been obtained using the analytical model and finite element method. Figure 5 shows the shape function i.e., $W(x)$, computed using the analytical method (see Figure 5 (a)) and finite element method (see Figure $5(\mathrm{~b}))$. The shape functions $(W(x))$ were chosen instead of the vibration mode shapes $(\mathrm{V}(\mathrm{x}))$ because it has effect of both the vibration mode shapes $(V(x))$ and the pitching motion $(\Theta)$, and hence it gives a better visualization of the physical scenario of a beam is undergoing transverse vibration as well as pitching motion. it is clear Figure 5 that the shape functions obtained using the analytical model and finite element method are identical. Thus, Figure5 along with Table 1validate the analytical method derived in the previous section.

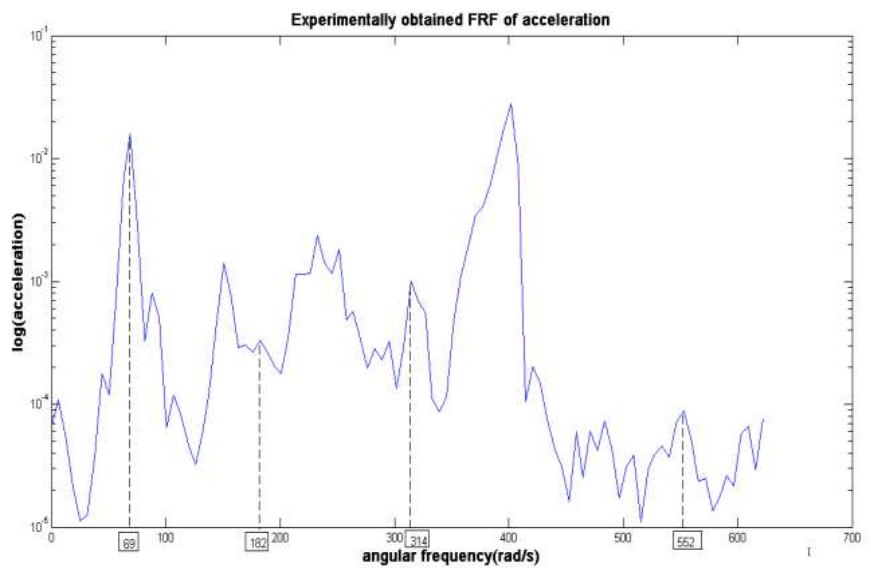

Figure 4. Experimentally obtained FRF of bounce acceleration.

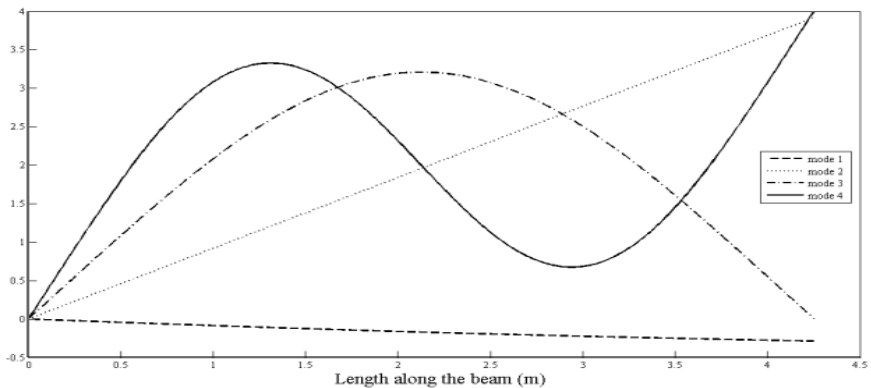

(a)

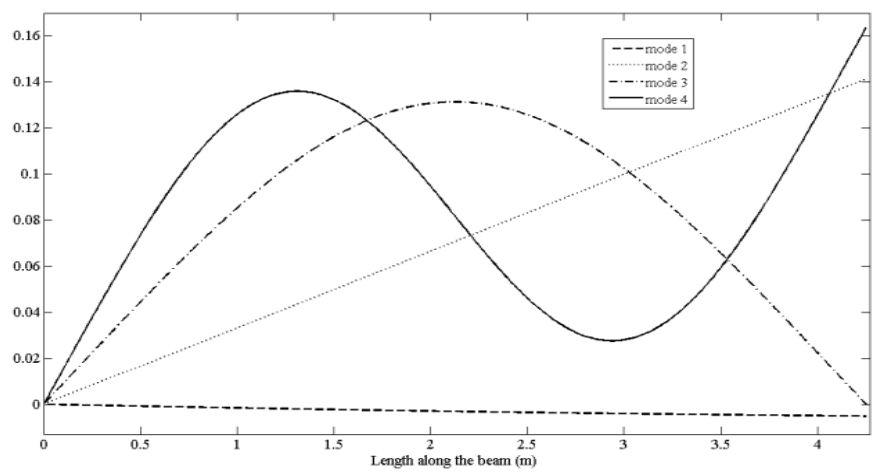

(b)

Figure 5. Shape-function $(\mathrm{W}(\mathrm{x}))$ along the length of the beam obtained using analytical method (a) and FEM (b). 
The impulse response function of a dynamic system can be used to compute the response of the dynamic system to any arbitrary excitation using the Duhamel's Integral [12]. Hence, to understand and compare the dynamic response of the models, it is important to analyze the unit impulse response of both the models. The simulation is run for 5 seconds and the first four modes of the flexible two dof model have been considered to compute the time response. Figure 6 shows the bounce displacement (in $\mathrm{m}$ ) and pitch displacement (in radian) for unit impulse excitation. It is clear from Figure 6 (a) that the rigid two dof model under predicts the bounce displacement (by more than 50\%) and has a lower settling time as compared to the flexible two dof model ( 30\%). Referring to Figure 6 (b), the rigid two dof model over predicts the pitch displacement $(\sim 50 \%)$ and has a higher settling time as compared to the flexible two dof model $(\sim 50 \%)$. The pitch response of the rigid two dof model is sluggish as compared to that of the flexible two dof model.

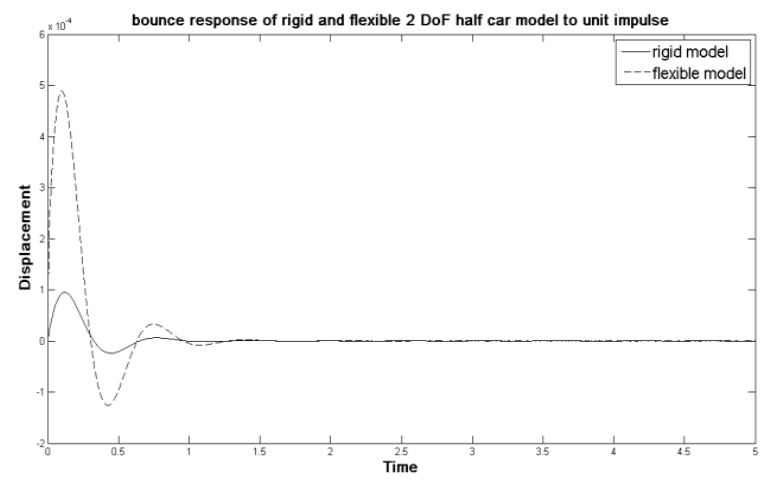

(a)

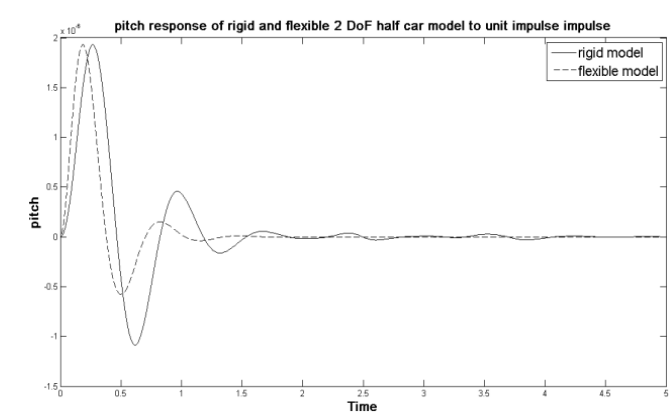

(b)

Figure 6. Bounce displacement (a) and pitch (b) response of the rigid two dof model and flexible two dof model to unit impulse.

It is a common practice in vehicle ride dynamics to study the response of the mathematical models to sinusoidal excitation. Road surface excitation is irregular/random, but it can be expressed as combination of sines and cosines with the help of Fourier Transform. Hence, it is essential to study the response of these models to sinusoidal base excitation. The front end is excited by a sinusoid of amplitude $0.01 \mathrm{~m}$ and angular frequency $\frac{\pi}{4} \mathrm{rad} / \mathrm{second}$. The rear end is excited by the same sinusoid but with a phase lag which can be computed using the length of the vehicle and velocity. The velocity is taken to be 1 $\mathrm{m} / \mathrm{s}$ and simulation is run for 30 seconds and the first 4 modes of the flexible two dof model have been considered.

It can be seen from Figure 7 (a) that the rigid two dof model predicts lower displacement amplitude as compared to the flexible two dof model. The flexible two dof model displacement plot (Figure 7 (a)) shows that the transmissibility is high because the output amplitude $(0.04 \mathrm{~m})$ is four times the input amplitude $(0.01 \mathrm{~m})$. Referring to Figure 7 (b), the pitch amplitude predicted by the rigid two dof model is lower than the flexible two dof model $(\sim 50 \%)$.

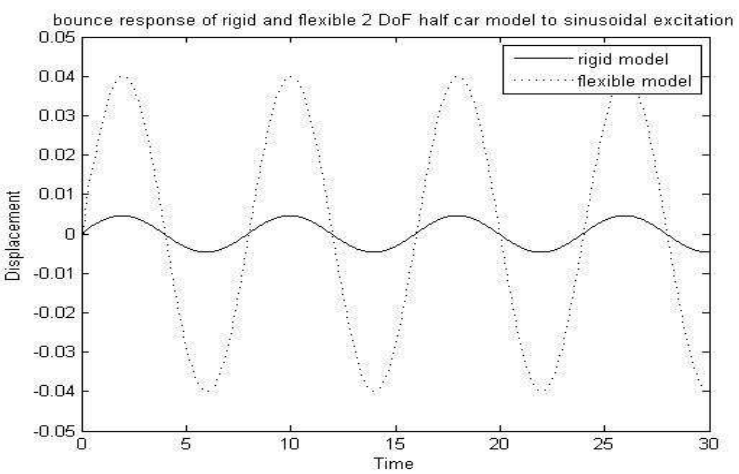

(a)

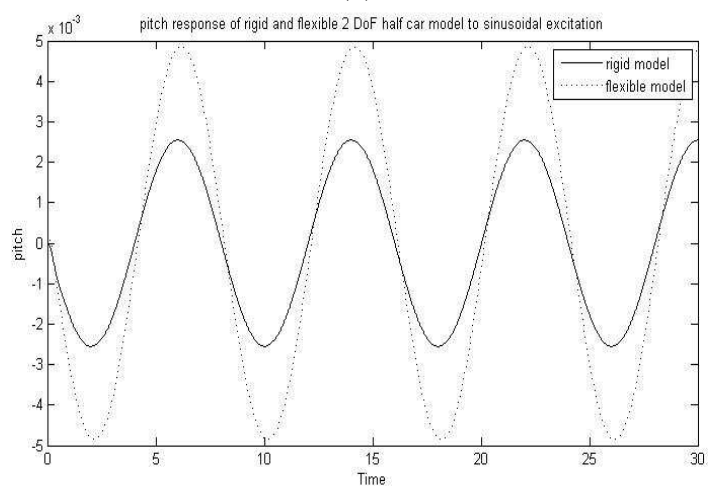

(b)

Figure7. Bounce displacement (a) and pitch (b) response of the rigid two dof model and flexible two dof model to sinusoidal excitation.

It can be concluded from Figure 6 and Figure 7 that there are significant differences in the time domain responses of the rigid two dof model and the flexible two dof model. Figure 8 shows the frequency response function (FRF) of the bounce displacement. The FRF of the rigid two dof model (Figure 8 (b)) has been computed by using the transfer function. The same method cannot be used for the flexible two dof model because of the non-linear $\mathbf{M}$ and $\mathbf{K}$ matrices. The FRF (Figure 
8 (a)) is obtained by solving the time domain system for a sinusoid of amplitude 1 and some angular frequency. The amplitude of the response is then calculated and plotted on the log-log plot corresponding to the angular frequency. 27 points were used to draw this FRF and these discrete points are then plotted to obtain an approximate sketch of the bounce displacement FRF of the flexible two dof model. The points associated with a box in this plot (see Figure $8(\mathrm{a})$ ) show the peaks corresponding to the four natural frequencies which are absent in the FRF of the rigid two dof model (see Figure 8 (b)). The amplitude ratio predicted by the rigid two dof model is lower than that of the flexible two dof model.

The additional modes of the system are responsible for the differences in dynamics of both the models. The flexible two dof model is closer to reality than the rigid two dof model and significant differences are observed between both the models, hence, the flexible two dof model should be used to do initial design calculations and control instead of the rigid two dof model.

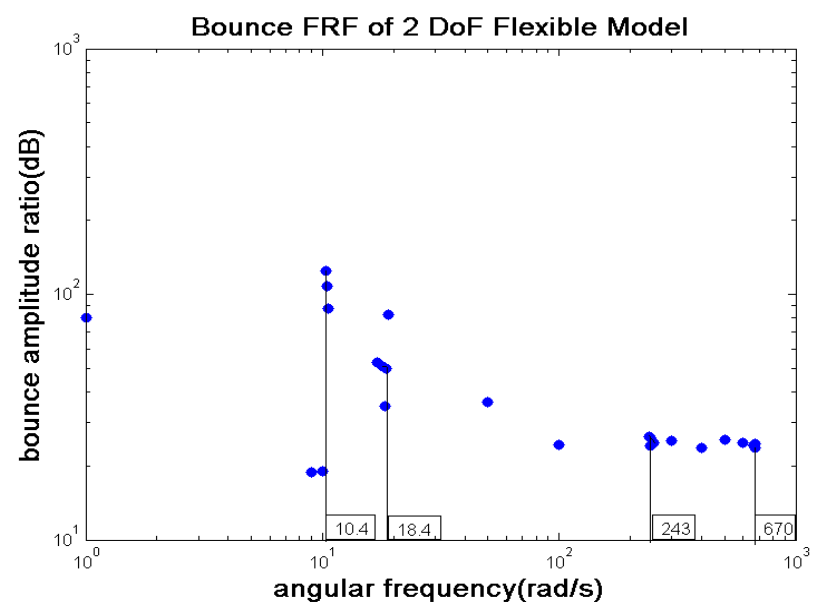

(a)

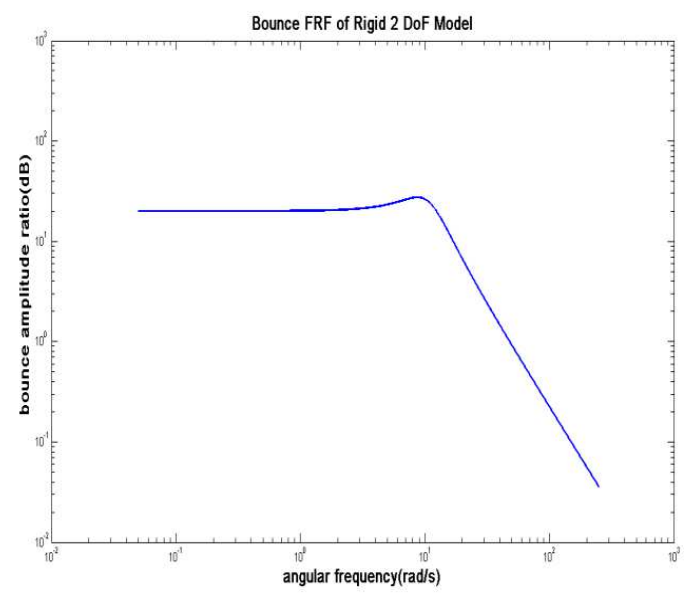

(b)

Figure 8. Frequency response of bounce of the flexible two dof model (a) and the rigid two dof model (b).

\section{SUMMARY}

A modified two dof model which accounts for the flexibility of the chassis by modeling it as a flexible beam is presented it in this paper. Complete set of the resulting dynamics equations are presented. The impulse response and sinusoidal response of the models are compared. The rigid two dof model under predicts the bounce displacement for both the excitation. The rigid two dof model shows a sluggish pitch response as compared to the flexible two dof model to unit impulse. The pitch amplitude predicted by the rigid two dof model is less than that predicted by the flexible two dof model to sinusoidal excitation. The settling time of the impulse response of bounce is lower for the rigid two dof model while for the pitch it is lower for the flexible two dof model. The bounce displacement amplitude ratio FRF of the flexible two dof model exhibits more peaks and higher amplitude ratio. The flexible two dof model is closer to reality as compared to the rigid two dof model, and hence should be used for initial design calculations, simulations and control design. An extension of the current work would be to develop a four dof vehicle ride model with sprung mass at the front and rear. Another work would be to account for tyre flexibility properly instead of considering it as a linear spring. It is important to note that only the first four have been used for simulations and the spring damper system are considered at the ends. The analytical model has been validated using FEM and experiments.

\section{REFERENCES}

1. Amirouche, Farid., Fundamentals of Multibody Dynamics, Birkhäuser, Boston, ISBN: 978-0-8176-42365,2006 .

2. Krysl, P., A Pragmatic Introduction to the Finite Element Method for Thermal and Stress Analysis, World Scientific Publishing Co. Pvt. Ltd., Singapore, ISBN-10: 981-256876-X | ISBN-13: 981-270-411-6, 2006.

3. Crolla, D.A., Vehicle Dynamics - Theory into Practice, Proceedings of the Institution of Mechanical Engineers, Part D: Journal of Automobile Engineering 210(2): 83-94, April 1996,doi:10.1243/PIME_PROC_1996_210_250_02.

4. Wong,J.Y., Theory of Ground Vehicles, John Wiley \& Sons, ISBN: 978-0-470-17038-0.

5. Sun, T., Zhang, Y. and Barak, P., 4-DOF Vehicle Ride Model, SAE Technical Paper 2002-01-1580, presented at SAE Automotive Dynamics and Stability Conference, Detroit, USA, May 2002.

6. Goncalves, J.P. and Ambrósio, J. A., Optimization of Vehicle suspension Systems for Improved Comfort of Road Vehicles Using Flexible Multibody Dynamics, Nonlinear Dynamics 34: 113-131, 2004.

7. Ibrahim, I.M., Crolla, D.A. and Barton, D.C., Effect of Frame Flexibility on the Ride Vibration of Trucks, Computers and Structures 58(4): 709-713, February 1996, doi:10.1016/0045-7949(95)00198-P. 
8. Verros, G. andNatsiavas, S., Ride Dynamics of Nonlinear Vehicle Models Using Component Mode Synthesis, Journal of Vibration and Acoustics 124(3): 427-434, July 2002,doi: $10.1115 / 1.1473828$.

9. Timoshenko, S.P. and Goodier, J.N., Theory of Elasticity, Tata McGraw-Hill Education Pvt. Ltd., New Delhi, ISBN-13: 978-0-07-070122-9, 2010.

10. Goldstein, H., Poole, C.P. and Safko, J., Classical Mechanics, Pearson, ISBN: 978-81-317-5891-5, June 2001.

11. Nayfeh,A. H., Emam,S. A., Preidikman,S., and Mook,D.T., An Exact Solution for the Natural Frequencies of Flexible Beams Undergoing Overall Motions, Journal of Vibration and Control 9(11): 12211229, November 2003, doi: 10.1177/1077546304030692.

12. Meirovitch, L., Analytical Methods in Vibration, Prentice Hall, New York, ISBN: 978-0023801402, 1967.

13. M. I. Friswell, John E. T. Penny, S. D. Garvey and Arthur W. Lees, Dynamics of Rotating Machines Book, Cambridge University Press, ISBN-10: 0521850169, 2010.

\section{LIST OF SYMBOLS}

$\mathrm{m}_{\mathrm{s}}$ sprung mass

$\mathrm{c}_{1}, \mathrm{c}_{2}$ damping coefficient of suspension

(1/f/11 : front and 2/r/22: rear)

$\mathrm{k}_{1}, \mathrm{k}_{2}$ suspension stiffness (1: front and

2: rear)

J

$\mathrm{X}_{\mathrm{rf}}, \mathrm{X}_{\mathrm{rr}}$ pitch moment of inertia

(f: front and r: rear)

$\mathrm{y}_{1}, \mathrm{y}_{2}$ road excitation input to flexible

model (1: front and 2: rear)

$\mathrm{X}_{\mathrm{S}}$

bounce displacement of rigid two

dof model

$\bar{y}$ bounce displacement of CG of

flexible two dof model

\section{$\bar{\theta}$}

pitch displacement of $\mathrm{CG}$ of

flexible two dof model

$\varphi$

pitch displacement of rigid two dof

model

$\mathrm{V}_{\mathrm{i}}(\mathrm{x}) \quad \mathrm{i}^{\text {th }}$ mode shape

$\eta_{\mathrm{i}}(\mathrm{t})$

time response of displacement in
$\mathrm{V}_{0}$

$1_{1}$

end

$1_{2}$

end

$\rho$

A

$\mathrm{E}$

material

I

chassis

L

$\mathrm{X}_{1}, \mathrm{X}_{2}$

ends (1:front and 2:rear) initial velocity of vehicle

distance of the CG from the front

distance of the $\mathrm{CG}$ from the rear

density of the chassis material

area of cross section of chassis

Young's Modulus of the chassis

area moment of inertia of the

length of chassis

distance of the suspension from the
ABBREVIATIONS

\begin{tabular}{|l|l|}
\hline Dof & Degree of freedom \\
\hline CG & Centre of gravity \\
\hline FRF & $\begin{array}{l}\text { Frequency Response } \\
\text { Function }\end{array}$ \\
\hline
\end{tabular}

\section{APPENDIX}

Rigid two dof model simulation parameters

Table 3. Physical parameters used in the simulations of the rigid two dof model

\begin{tabular}{|l|l|}
\hline Sprung mass $\left(m_{\mathrm{s}}\right)$ & $603.043 \mathrm{~kg}$ \\
\hline Front suspension stiffness $\left(k_{1}\right)$ & $35000 \mathrm{~N} / \mathrm{m}$ \\
\hline Rear suspension stiffness $\left(k_{1}\right)$ & $32000 \mathrm{~N} / \mathrm{m}$ \\
\hline Distance of CG from front $\left(l_{1}\right)$ & $2.125 \mathrm{~m}$ \\
\hline Distance of CG from rear $\left(l_{2}\right)$ & $2.125 \mathrm{~m}$ \\
\hline Front damping coefficient $\left(c_{1}\right)$ & $2570 \mathrm{Ns} / \mathrm{m}$ \\
\hline Rear damping coefficient $\left(c_{2}\right)$ & $2480 \mathrm{Ns} / \mathrm{m}^{2}$ \\
\hline Moment of inertia $(J)$ & $3630.84 \mathrm{kgm}$ \\
\hline
\end{tabular}

body fixed frame due to $i^{\text {th }}$ mode 
Flexible two dof model simulation parameters

Table 4. Physical parameters used in the simulations of the flexible two dof model

\begin{tabular}{|l|l|}
\hline Sprung mass $\left(m_{\mathrm{s}}\right)$ & $603.043 \mathrm{~kg}$ \\
\hline Front suspension stiffness $\left(k_{1}\right)$ & $35000 \mathrm{~N} / \mathrm{m}$ \\
\hline Rear suspension stiffness $\left(k_{2}\right)$ & $32000 \mathrm{~N} / \mathrm{m}$ \\
\hline Distance of CG from front $\left(l_{1}\right)$ & $2.125 \mathrm{~m}$ \\
\hline Distance of CG from rear $\left(l_{2}\right)$ & $2.125 \mathrm{~m}$ \\
\hline Front damping coefficient $\left(c_{1}\right)$ & $2570 \mathrm{Ns} / \mathrm{m}$ \\
\hline Rear damping coefficient $\left(c_{2}\right)$ & $2480 \mathrm{Ns} / \mathrm{m}$ \\
\hline Moment of inertia $\left(J_{0}\right)$ & $3630.84 \mathrm{kgm}$ \\
\hline Young's Modulus $(\mathrm{E})$ & $210 \mathrm{GPa}$ \\
\hline Area moment of inertia, $(\mathrm{I})$ & $2.6 X 10^{-5} \mathrm{~m}^{4}$ \\
\hline Density, $\rho$ & $7850 \mathrm{~kg} / \mathrm{m}^{3}$ \\
\hline$x_{1} \& x_{2}$ & $0 \mathrm{~m}$ \\
\hline
\end{tabular}

Experimental setup parameters

Table 5. Physical parameters of the experimental setup

\begin{tabular}{|l|l|}
\hline Sprung mass $\left(m_{\mathrm{s}}\right)$ & $1.7786 \mathrm{~kg}$ \\
\hline Front suspension stiffness $\left(k_{1}\right)$ & $13757 \mathrm{~N} / \mathrm{m}$ \\
\hline Rear suspension stiffness $\left(k_{2}\right)$ & $13757 \mathrm{~N} / \mathrm{m}$ \\
\hline Distance of CG from front $\left(l_{1}\right)$ & $0.5125 \mathrm{~m}$ \\
\hline Distance of CG from rear $\left(l_{2}\right)$ & $0.5125 \mathrm{~m}$ \\
\hline Moment of inertia $\left(J_{0}\right)$ & $0.1557 \mathrm{kgm}^{2}$ \\
\hline Young's Modulus $(\mathrm{E})$ & $210 \mathrm{GPa}$ \\
\hline Area moment of inertia, $(\mathrm{I})$ & $5.57 \mathrm{X} 10^{-10} \mathrm{~m}^{4}$ \\
\hline Density, $(\rho)$ & $7850 \mathrm{~kg} / \mathrm{m}^{3}$ \\
\hline$x_{1}$ and $x_{2}$ & $0 \mathrm{~m}$ \\
\hline
\end{tabular}

